hep-th/0602103

ITFA-2006-06

\title{
On the critical points of the entropic principle
}

\author{
Bartomeu Fiol \\ Institute for Theoretical Physics, University of Amsterdam \\ 1018 XE Amsterdam, The Netherlands \\ bfiol@science.uva.nl
}

\begin{abstract}
In a recent paper, hep-th/0509109, Gukov et al. introduced an entropy functional on the moduli space of Calabi-Yau compactifications. The maxima of this functional are then interpreted as 'preferred' Calabi-Yau compactifications. In this note we show that for compact Calabi-Yaus, all regular critical points of this entropic principle are maxima.
\end{abstract}




\section{Are some vacua more equal than others?}

A feature of $\mathrm{M} /$ string theory that is receiving increasing attention is the vast number of vacua, even if we decide to restrict ourselves to static solutions with $3+1$ macroscopic dimensions and $\mathcal{N}=0,1$ (the papers devoted to the subject constitute a landscape on their own; see e.g. 1 for reviews). Faced with this plethora of solutions, some natural questions are whether there is a natural measure on the space of solutions, and whether there is any built-in mechanism in $\mathrm{M} /$ string theory that favors some vacua over others.

A possible way of assigning weights to vacua is to consider a Wheeler-deWitt equation [2] for families of vacua of string theory. This defines a quantum mechanical problem over some space of solutions, and the modulus square of the wavefunction is interpreted as a probability distribution for the different solutions.

For $4 \mathrm{~d}$ flux vacua with $\mathcal{N}=0,1$, the Wheeler-deWitt equation has been discussed in [3] ${ }^{1}$. A slightly different scenario for flux vacua was considered in [5], where compactifications of type IIB string theory on $S^{1} \times S^{2} \times C Y$ were studied. For each choice of flux, a wavefunction $\Psi_{(p, q)}$ was introduced, and using the intimate connection between flux vacua and the attractor mechanism, it was then argued that the peaks of the different wavefunctions are given by the exponentials of the entropies of related black holes with charges $(p, q)$.

In this scenario, it is natural to ask which flux vacuum has a wavefunction with the largest peak, which semiclassically is equivalent to maximizing the exponentiated Kähler potential

$$
S=-\frac{i \pi}{4} \int_{M} \Omega \wedge \bar{\Omega}
$$

over the complex structure moduli space of the Calabi-Yau. However, this question is not well posed as stated, since this functional is not invariant under Kähler gauge transformations. Physically, this is related to the fact that a rescaling of the charges of the black hole leaves invariant the attractor point, but rescales the entropy. To fix this rescaling ambiguity, the authors of [6] propose to extremize this functional subject to the condition that we hold fixed one of the periods,

$$
\int_{C} \Omega=1
$$

for some 3 -cycle $C$. In terms of black holes, this amounts, via the attractor equations, to scan only over black holes with one of their charges fixed at a particular value. The entropic principle claims that the maximum of this action, subjected to the constraint of fixing one period, constitute the preferred Calabi-Yau.

\footnotetext{
${ }^{1}$ see [4] for a discussion of the Wheeler-deWitt equation for M-theory compactifications preserving 32 and 16 supersymmetries.
} 
In this way of presenting the principle, the choice of period to be fixed seems quite arbitrary. The authors of [6] argue that an equivalent formulation of their principle consists in finding the points in Calabi-Yau moduli space where all the periods but one are aligned. To decide if a critical point is a maximum, saddle point or minimum, we need to compute the second variation of the functional. The character of the critical point is then determined by the signature of a reduced period matrix, $\operatorname{Im} \tau_{i j}$. One might have hoped that in the best possible scenario, for a given Calabi-Yau moduli space, of all such critical points, only one or very few are actually maxima.

In this note we argue that at the critical points of compact Calabi-Yaus, the reduced period matrix always satisfies $\operatorname{Im} \tau_{i j}>0^{2}$. This implies that all critical points are maxima. Since for a given Calabi-Yau moduli space one expects an infinite number of critical points (due to the possibility of fixing infinitely many different periods), our result shows that the entropic principle will need extra input as to why any particular period should be fixed, in order to be useful in selecting a single point in Calabi-Yau moduli space.

In the next section we give a brief review of the construction of wavefunctions for string vacua, and the entropic principle of [6]. We then proceed to show our main result, namely that all critical points satisfy $\operatorname{Im} \tau_{i j}>0$. A simple family of examples is considered in the last section.

\section{Wavefunctions for flux vacua and the entropic principle}

In this section we start by recalling the basics of the special geometry of the complex structure moduli space of a Calabi-Yau [7, 8]. After reviewing the construction of wavefunctions of [5] and the proposal of an entropic principle of [6], we show that all critical points of this extremization problem are actually maxima.

\subsection{Special geometry}

Given a compact Calabi-Yau $M$, we define the Hodge-Riemann bilinear form [9] on its cohomology groups, $Q: H^{3-k} \otimes H^{3-k} \rightarrow \mathbb{C}$ by

$$
Q(\alpha, \beta)=\int_{M} \alpha \wedge \beta \wedge J^{k}
$$

\footnotetext{
${ }^{2}$ The only possible exception being if $\operatorname{Im} \tau_{i j}$ has one or more zero eigenvalues.
} 
The Hodge-Riemann relations assert that if $\alpha$ is a primitive $(p, q)$ form, then ${ }^{3}$

$$
i^{p-q}(-1)^{\frac{(3-p-q)(2-p-q)}{2}} Q(\alpha, \bar{\alpha})>0
$$

With these conventions, the exponentiated Kähler potential (multiplied here by a convenient constant) is semipositive

$$
S=\frac{\pi}{4} e^{-K_{c s}}=i^{3} \frac{\pi}{4} \int_{M} \Omega \wedge \bar{\Omega}
$$

Let $\left\{A^{I}, B_{I}\right\}, I=0, \ldots, h^{2,1}(M)$ be a symplectic basis for $H_{3}(M, \mathbb{Z})$, and $\left(\alpha_{I}, \beta^{I}\right)$ their Poincaré dual forms $\left(\alpha_{I}, \beta^{I}\right)$. In this basis, the periods are

$$
X^{I}=\int_{A^{I}} \Omega \quad F_{I}=\int_{B_{I}} \Omega
$$

One can argue that the periods $F_{I}$ derive from a prepotential $F_{I}=\partial_{I} \mathcal{F}$, with $\mathcal{F}(X)$ a homogeneous function of degree two. In terms of the periods,

$$
S=\frac{\pi}{4} e^{-K_{c s}}=\frac{i \pi}{4}\left\{\bar{X}^{I} F_{I}-X^{I} \bar{F}_{I}\right\}
$$

Finally, define the period matrix as $\tau_{I J}=\frac{\partial F_{J}}{\partial X^{I}}$. Since $\partial_{I} \Omega=\alpha_{I}-\tau_{I J} \beta^{J}$, it follows that

$$
\operatorname{Im} \tau_{I J}=\frac{i}{2} \int_{M} \frac{\partial \Omega}{\partial X^{I}} \wedge \overline{\frac{\partial \Omega}{\partial X^{J}}}
$$

Now, using that $\partial_{I} \Omega \in H^{3,0} \oplus H^{2,1}$, and applying the Hodge-Riemann relations we learn that $\operatorname{Im} \tau_{I J}$ has signature $\left(1, h^{21}\right)^{4}$.

The periods provide projective coordinates for the moduli space, but it will be convenient to introduce affine coordinates $a^{i}=X^{i} / X^{0}, i=1, \ldots, h^{2,1}(M)$ and define $F(a)=\left(X^{0}\right)^{-2} \mathcal{F}(X), a_{i}^{D}=\frac{\partial F(a)}{\partial a^{i}}$. The action can be rewritten in terms of these coordinates as

$$
S=\frac{i \pi}{4}\left|X^{0}\right|^{2}\left\{2(F-\bar{F})-\left(a^{i}-\bar{a}^{i}\right)\left(a_{i}^{D}+\bar{a}_{i}^{D}\right)\right\}
$$

\footnotetext{
${ }^{3}$ The overall sign of the Hodge-Riemann bilinear form fluctuates in the literature, due to different choices of orientation. We follow the conventions of $[9]$.

${ }^{4}$ The overall sign convention mentioned in the previous footnote would show up also here. The result that is independent of conventions is that for an exponentiated Kähler potential that is real and semipositive definite, its matrix of second derivatives (- $\operatorname{Im} \tau_{I J}$ in our conventions) has signature $\left(1, h^{21}\right)$, where the first entry denotes the number of negative eigenvalues.
} 


\subsection{Wavefunctions for compactifications without fluxes.}

There are some obvious similarities between the period matrix $\operatorname{Im} \tau_{I J}$ and the deWitt metric: both are defined over a space of space-like metrics, and both have hyperbolic signature, with the 'timelike' direction reflecting the possibility of rescaling the spacelike metrics. It is then natural to consider whether there is a minisuperspace approach to Calabi-Yau compactifications, where $\operatorname{Im} \tau_{I J}$ plays the role of metric in superspace. String theory provides a very natural candidate for a quantum mechanical system defined over $H^{3}(M)$ : the B-model topological string. The first hint is that the operators of the B-model topological string are in one to one correspondence with the $(2,1)$ cohomology of the Calabi-Yau. More importantly, it was argued in [10] that the B-model topological string partition function is a wavefunction on $H^{3}(M)$. This partition function satisfies the holomorphic anomaly equations [11. These holomorphic anomaly equations can be written in terms of large phase space variables [12, 13, with Im $\tau_{I J}$ playing the role of metric in this large phase space. The holomorphic anomaly equations, however, should not be thought of as the Wheeler-deWitt equation for the topological string: they only reflect the background dependence on the choice of polarization $^{5}$. It would be interesting to understand what wave equation plays the role of Schrödinger equation for the topological string partition function.

\subsection{Wavefunctions for flux vacua.}

Another family of string compactifications where wavefunctions can be computed was discussed in [5]. They consider type IIB with all spatial dimensions compactified, on $S^{1} \times S^{2} \times M$, with $M$ a Calabi-Yau, and fluxes turned on. More specifically, a $F_{5}$ flux of the form $w \wedge F_{3}$ is turned on, where $w$ is a unit form on $S^{2}$ and $F_{3}$ is a RR form determined by the fluxes $\left(p^{I}, q_{I}\right)$,

$$
p^{I}=\int_{A^{I}} F_{3} \quad q_{I}=\int_{B^{I}} F_{3}
$$

The conditions for this flux to preserve supersymmetry are intimately related to the attractor equations of a different system: that of the compactification on $M$ with wrapped D3-branes with charges $\left(p^{I}, q_{I}\right)$. The attractor mechanism fixes a point in the complex structure moduli of $M$ in terms of the charges $\left(p^{I}, q_{I}\right)$

$$
p^{I}=\operatorname{Re}\left(C X^{I}\right) \quad q_{I}=\operatorname{Re}\left(C F_{I}\right)
$$

For a fixed choice of $\left(p^{I}, q_{I}\right)$, [5] define the entropy functional

$$
S_{(p, q)}=-i \frac{\pi}{4}\left(\int \Omega \wedge \bar{\Omega}+\int(\Omega+\bar{\Omega}) \wedge F_{3}\right)
$$

\footnotetext{
${ }^{5}$ I would like to thank R. Dijkgraaf and K. Skenderis for useful comments on this point.
} 
This functional has two nice properties: first, when we extremize it with respect to $X^{I}$, the equations of motion are the attractor equations, for $C=1$. Second, when this functional is evaluated at the solution, it gives the semiclassical entropy of a black hole of charges $\left(p^{I}, q_{I}\right)$,

$$
\left.S_{(p, q)}\right|_{a t t}=S_{B H}\left(p^{I}, q_{I}\right)
$$

The authors of [5] promote the classical BPS equations for the attractor flows to a supersymmetric version of the Wheeler-deWitt equation. In this fashion, for each choice of fluxes $(p, q)$, one has a wavefunction $\Psi_{(p, q)}$. These wavefunctions are peaked at the attractor point, and furthermore it can be argued [5] that their natural normalization is such that the value at the peak behaves like $e^{S_{B H}(p, q)}$.

\subsection{The entropic principle}

If we repeat this process for very many different charge vectors $\left(p^{I}, q_{I}\right)$, we will find many attractor points in moduli space, and we might be tempted to assign to each of them the weight $e^{S_{B H}\left(p^{I}, q_{I}\right)}$. However, this assignment is not well defined as it stands: if we consider a set or rescaled charges $\left(\lambda p^{I}, \lambda q_{I}\right)$, the solution to the attractor equations is still the same point in moduli space (recall the $X^{I}$ are projective coordinates), but the entropy of the corresponding black hole has changed. If we want to unambiguously assign a weight to a given point in the Calabi-Yau moduli space, we first need to fix this ambiguity in the rescaling of the charges. A proposal for how to do this was presented in [6] to which we now turn.

Consider the flux independent part of the entropy functional

$$
S=i^{3} \frac{\pi}{4} \int_{M} \Omega \wedge \bar{\Omega}
$$

This functional evaluated at an attractor point still gives the semiclassical entropy of the corresponding black hole, so the first thought might be trying to maximize it. However, as we have just seen, as far as determining attractor points in moduli space, the charge lattice behaves like a projective variety: $\left(\lambda p^{I}, \lambda q_{I}\right)$ yield the same attractor point that $\left(p^{I}, q_{I}\right)$. A possibility is then to fix a hypersurface in this charge space, e.g. fix one of the charges. In particular, in [6] they propose to extremize the functional $S$, keeping fixed one period $X^{c}$. Since we are fixing the period of a 3-cycle, without loss of generality we take that cycle to be primitive. Now, since the action of the symplectic group is transitive, we can always take that primitive cycle to be an A-cycle in a symplectic basis. So without loss of generality, we can fix the period $X^{0}$. For this purpose, one first rewrites this entropy functional in terms of the "reduced" periods 
$a^{i}=X^{i} / X^{0}, a_{i}^{D}$

$$
S=\frac{i \pi}{4}\left|X^{0}\right|^{2}\left\{2(F-\bar{F})-\left(a^{i}-\bar{a}^{i}\right)\left(a_{i}^{D}+\bar{a}_{i}^{D}\right)\right\}
$$

At the attractor point, this is equivalent to fixing a magnetic charge $p^{0}$ and $\phi^{0}$, the chemical potential of its dual electric charge 14. In this way we get rid of the ambiguity in assigning an entropy to points in moduli space, and comparing the different entropies becomes well-defined.

This entropy functional has critical points with respect to the variation of $a^{i}, \bar{a}^{i}$, given by the equation

$$
\operatorname{Im} a_{i}^{D}-\tau_{i j} \operatorname{Im} a^{j}=0
$$

Since the signature of $\operatorname{Im} \tau_{I J}$ is $\left(1, h^{2,1}\right)$, the signature of $\operatorname{Im} \tau_{i j}$ is either $\left(0, h^{2,1}\right)$ or $\left(1, h^{2,1}-1\right)$. Furthermore, taking the imaginary part of this equation, we see that if $\left|\operatorname{Im} \tau_{i j}\right| \neq 0$, all critical points have $\operatorname{Im} a^{i}=0$. To determine if these critical points are maxima, minima or saddle points, we need to consider the matrix of second derivatives

$$
\delta^{2} S=-\pi\left|X^{0}\right|^{2}\left(\operatorname{Im} \tau_{i j}\right) \delta a^{i} \delta \bar{a}^{j}+\frac{\pi}{2}\left|X^{0}\right|^{2} \operatorname{Im} a^{i}\left(c_{i j k} \delta a^{j} \delta a^{k}+\bar{c}_{i j k} \delta \bar{a}^{j} \delta \bar{a}^{k}\right)
$$

with $c_{i j k}=\partial_{i} \tau_{j k}$. Since $c_{i j k} \operatorname{Im} a^{k}$ does not have a definite signature, if it is different from zero, the critical point can't be a maximum. A sufficient condition for $c_{i j k} \operatorname{Im} a^{k}=0$ at a critical point is that $\left|\operatorname{Im} \tau_{i j}\right| \neq 0$, since then $\operatorname{Im} a^{i}=0$. Therefore, if $\operatorname{Im} \tau_{i j}>0$ the critical point is a maximum, and if $\operatorname{Im} \tau$ has hyperbolic signature, the critical point is a saddle point (or a minimum if $h^{2,1}=1$ ). Maxima are then of the form

$$
\operatorname{Im} a^{i}=\operatorname{Im} a_{i}^{D}=0 \quad \operatorname{Im} \tau_{i j}>0
$$

Such a point in moduli space solves the attractor equations for $p^{i}=q_{i}=0$. This solution allows for an alternative reformulation of the principle [6]: the critical points in Calabi-Yau moduli space are such that all the periods but one are aligned. Then, the 3-cycle whose period is fixed is the symplectic dual to the 3-cycle that has null intersection with all the 3-cycles with aligned periods.

We now will give two arguments showing that at critical points it is always the case that $\operatorname{Im} \tau_{i j} \geq 0$, with the equality only possible if furthermore $\partial_{i} \Omega=0$ for some $i$. Otherwise, we have a maximum. The first argument is quite simple. Using that $X^{0}$ is fixed, we can write the imaginary part of the reduced period matrix as

$$
\operatorname{Im} \tau_{i j}=\frac{i}{2\left|X^{0}\right|^{2}} \int_{M} \frac{\partial \Omega}{\partial a^{i}} \wedge \frac{\overline{\partial \Omega}}{\partial a^{j}}
$$

By Griffiths transversality, we know that $\partial_{i} \Omega \in H^{3,0} \oplus H^{2,1}$. Specifically [8],

$$
\frac{\partial \Omega}{\partial a^{i}}=-\frac{\partial K}{\partial a^{i}} \Omega+D_{i} \Omega
$$


But since $S=\frac{\pi}{4} e^{-K}$, the equation for critical point $\partial_{i} S=0$ is equivalent to $\partial_{i} K=0$ unless $K= \pm \infty$. So, at critical points $\partial_{i} \Omega \in H^{2,1}$, and it then follows from the Hodge-Riemann relations that at a critical point $\operatorname{Im} \tau_{i j}>0$.

We now give a slightly different proof. Starting with the the definition $F(a)=$ $\left(X^{0}\right)^{-2} \mathcal{F}(X)$, we can write the second derivatives of the full prepotential in terms of reduced quantities

$$
\frac{\partial^{2} \mathcal{F}}{\partial X^{I} \partial X^{J}}=\left(\begin{array}{cc}
2 F-2 a^{i} \partial_{i} F+a^{i} \tau_{i j} a^{j} & \partial_{i} F-a^{j} \tau_{j i} \\
\partial_{i} F-a^{j} \tau_{j i} & \tau_{i j}
\end{array}\right)
$$

If we now consider the imaginary part of this equation and take determinants, we arrive at the relation

$$
\left|\operatorname{Im} \tau_{I J}\right|=-\frac{2 S}{\pi\left|X^{0}\right|^{2}}\left|\operatorname{Im} \tau_{i j}+\frac{2}{\pi\left|X^{0}\right|^{2}} \frac{\operatorname{Re} \partial_{i} S \operatorname{Re} \partial_{j} S}{S}\right|
$$

At a critical point, this yields

$$
\left|\operatorname{Im} \tau_{I J}\right|_{\text {crit }}=-\frac{2 S}{\pi\left|X^{0}\right|^{2}}\left|\operatorname{Im} \tau_{i j}\right|_{\text {crit }}
$$

We know that $\left|\operatorname{Im} \tau_{I J}\right|<0$ and $S>0$, even away from critical points, so it follows that $\left|\operatorname{Im} \tau_{i j}\right|_{\text {crit }}>0$. Since the only two possibilities were that $\operatorname{Im} \tau_{i j}>0$ or that $\operatorname{Im} \tau_{i j}$ had hyperbolic signature, we again conclude that all critical points have $\operatorname{Im} \tau_{i j}>0$.

Some remarks are in order. First, we have carried out the discussion exclusively in the complex structure moduli space. One can adapt the discussion to the complexified Kähler moduli space, and in fact, an equation very similar to (2.21) appeared already in [8] (see the last equation in their section 4), valid in the interior of the complexified Kähler cone, and when one fixes the period of the 0-cycle. However, a crucial difference is that in that particular case, the reduced period matrix has always hyperbolic signature: this is ultimately due to the fact that the Hodge-Riemann form has definite sign on $H^{2,1}$, while on $H^{1,1}$ has hyperbolic signature, since it differentiates the Kähler class from primitive forms. This does not conflict with mirror symmetry, since mirror symmetry between Calabi-Yau manifolds $M$ and $W$ does not exchange the Hodge-Riemann form on $H^{2,1}(M)$ with that on $H^{1,1}(W)$.

Secondly, our discussion was restricted to compact Calabi-Yaus. For non-compact Calabi-Yaus, all the periods can actually be aligned at some locus of the moduli space: this has been studied for orbifolds [15], ALE fibrations [16], and line bundles over del Pezzo surfaces [17. However, when zooming in a compact Calabi-Yau into a noncompact one, some periods become infinite, and there is no symplectic basis in general. The results we derived do not necessarily apply in the non-compact case, but we see this as an indication of the limitations of studying non-compact Calabi-Yaus, which at any rate are not our ultimate interest. 


\section{Some examples of critical points}

As an illustration of our results, we will revisit the case of the large complex structure limit of one-modulus models, already discussed in [6]. There, an infinite number of critical points were identified, and it was further claimed that there were minima of the entropic principle. We argue here that, in accordance with our general results, those critical points are actually maxima.

The prepotential for these models in the large complex structure limit is

$$
\mathcal{F}=-\frac{1}{3} \frac{\left(X^{1}\right)^{3}}{X^{0}}
$$

In [6], they focused on the subspace with $\operatorname{Im} X^{0}=\operatorname{Re} X^{1}=0$, and noted that points where

$$
\left(\frac{X^{1}}{X^{0}}\right)^{2}=n
$$

for some negative integer $n$ are critical points. To ease the notation, it is convenient to add a quadratic term to the prepotential

$$
\mathcal{F}=-\frac{1}{3} \frac{\left(X^{1}\right)^{3}}{X^{0}}+n X^{0} X^{1}
$$

This is equivalent to the $S L(4, \mathbb{Z})$ transformation performed in $[6]$. The periods are then

$$
\left(\begin{array}{c}
X^{0} \\
X^{1} \\
F_{1} \\
F_{0}
\end{array}\right)=\left(\begin{array}{c}
X^{0} \\
X^{1} \\
-\frac{\left(X^{1}\right)^{2}}{X^{0}}+n X^{0} \\
\frac{1}{3} \frac{\left(X^{1}\right)^{3}}{\left(X^{0}\right)^{2}}+n X^{1}
\end{array}\right)
$$

Now, as pointed out in [6], whenever $\left(X^{1} / X^{0}\right)^{2}=n$, the $F_{1}$ period vanishes, so the periods $\left(X^{1}, F_{1}, F_{0}\right)$ align, while $X^{0}$ is not aligned with them. The non-aligned period is symplectically dual to the fixed one [6], so the fixed period is $F_{0}$, which is a magnetic (B-cycle) period with respect to the prepotential. To write down the action (which of course is symplectically invariant) in terms of reduced variables we used that the prepotential is homogeneous in terms of the A-periods, and all the subsequent discussion of full vs. reduced period matrix is based on a given choice of symplectic basis. Therefore, before we introduce reduced periods by fixing $F_{0}$, we perform a symplectic transformation so $F_{0}$ becomes an electric (A-cycle) period,

$$
\left(\begin{array}{c}
\tilde{X}^{0} \\
\tilde{X}^{1} \\
\tilde{F}_{1} \\
\tilde{F}_{0}
\end{array}\right)=\left(\begin{array}{c}
F_{0} \\
F_{1} \\
-X^{1} \\
-X^{0}
\end{array}\right)
$$


and the new prepotential is $\tilde{\mathcal{F}}=\mathcal{F}-\left(X^{I} F_{I}\right)=-\mathcal{F}$, which differs by a minus sign from the one used in [6]. In these dual variables we can now define the usual reduced variables, starting with $\tilde{F}=\left(\tilde{X}^{0}\right)^{-2} \tilde{\mathcal{F}}$. Straightforward computation at a critical point $\left(X^{1} / X^{0}\right)^{2}=n$ then yields

$$
\operatorname{Im} \tilde{\tau}=\frac{1}{4 \sqrt{|n|}}>0
$$

so these points are maxima, as they had to be according to our general arguments.

Acknowledgments. I would like to thank Sergei Gukov, Kirill Saraikin and Cumrun Vafa for correspondence on [6] and the present paper. I have benefited from insightful comments by Jan de Boer, Robbert Dijkgraaf, Lotte Hollands and Kostas Skenderis. Special thanks to Erik Verlinde for many discussions and explanations on these matters. I would also like to thank the theory groups at the Universidad Autónoma de Madrid and the Universitat de Barcelona for giving me the chance to present related material at their respective Xmas. Workshops. Finally, during the course of this work, I greatly enjoyed the hospitality of the theory groups (and the pastry chef) at CERN and at the Universidade de Santiago de Compostela.

\section{References}

[1] M. R. Douglas, Comptes Rendus Physique 5, 965 (2004) arXiv:hep-th/0409207. J. Kumar, arXiv:hep-th/0601053.

[2] B. S. DeWitt, Phys. Rev. 160, 1113 (1967).

[3] A. Kobakhidze and L. Mersini-Houghton, arXiv:hep-th/0410213. L. MersiniHoughton, Class. Quant. Grav. 22, 3481 (2005) arXiv:hep-th/0504026. S. Sarangi and S. H. Tye, arXiv:hep-th/0505104. R. Brustein and S. P. de Alwis, arXiv:hep-th/0511093.

[4] T. Banks, W. Fischler and L. Motl, JHEP 9901, 019 (1999) arXiv:hep-th/9811194. L. Motl and T. Banks, JHEP 9905, 015 (1999) arXiv:hep-th/9904008.

[5] H. Ooguri, C. Vafa and E. P. Verlinde, arXiv:hep-th/0502211.

[6] S. Gukov, K. Saraikin and C. Vafa, arXiv:hep-th/0509109.

[7] A. Strominger, Commun. Math. Phys. 133 (1990) 163.

[8] P. Candelas and X. de la Ossa, Nucl. Phys. B 355, 455 (1991).

[9] Principles of Algebraic Geometry. P. Griffiths and J. Harris. Wiley Interscience.

[10] E. Witten, arXiv:hep-th/9306122.

[11] M. Bershadsky, S. Cecotti, H. Ooguri and C. Vafa, Commun. Math. Phys. 165, 311 (1994) arXiv:hep-th/9309140.

[12] R. Dijkgraaf, E. P. Verlinde and M. Vonk, arXiv:hep-th/0205281.

[13] E. P. Verlinde, arXiv:hep-th/0412139. 
[14] H. Ooguri, A. Strominger and C. Vafa, Phys. Rev. D 70, 106007 (2004) arXiv:hep-th/0405146.

[15] M. R. Douglas, B. R. Greene and D. R. Morrison, Nucl. Phys. B 506, 84 (1997) arXiv:hep-th/9704151. D. E. Diaconescu and J. Gomis, JHEP 0010, 001 (2000) arXiv:hep-th/9906242. M. R. Douglas, B. Fiol and C. Römelsberger, JHEP 0509, 057 (2005) arXiv:hep-th/0003263.

[16] B. Fiol, arXiv:hep-th/0012079.

[17] P. S. Aspinwall and I. V. Melnikov, JHEP 0412, 042 (2004) arXiv:hep-th/0405134. 